\title{
Dimensionality Reduction of Optical Coherence Tomography Images for the Early Diagnosis of Alzheimer's Disease
}

\author{
Sandeep C S $^{1, *}$, Sukesh Kumar A ${ }^{1}, \mathbf{K}$ Mahadevan², Manoj $\mathbf{P}^{3}$ \\ ${ }^{1}$ Department of Electronics and Communication, College of Engineering, Trivandrum, India \\ ${ }^{2}$ Department of Ophthalmology, Sree Gokulam Medical College and Research Foundation, Trivandrum, India \\ ${ }^{3}$ Department of Neurology, Sree Gokulam Medical College and Research Foundation, Trivandrum, India \\ *Corresponding author: sandeepcs07nta@gmail.com
}

\begin{abstract}
Alzheimer's disease (AD) is the most common cause of dementia and its incidence is increasing worldwide along with population aging. Previous clinical and histologic studies suggest that the neurodegenerative process, which affects the brain, may also affect the retina of $\mathrm{AD}$ patients. In this case, the employment of advanced Biomedical Engineering Technology will definitely helpful for making diagnosis. Any disease modifying treatments which are developed are most possibly to be achieving success if initiated early in the process, and this needs that we tend to develop reliable, validated and economical ways to diagnose Alzheimer's kind pathology. However, despite comprehensive searches, no single test has shown adequate sensitivity and specificity, and it is likely that a combination will be needed. Profiling of human body parameter using computers can be utilised for the early Diagnosis of Alzheimer's disease. There are lot of tests and imaging modalities to be performed for an effective Diagnosis of the disease. Prominent of them are Magnetic Resonance Imaging Scan (MRI), Positron Emission Tomography (PET), Single Photon Emission CT Scanning (SPECT) and Optical Coherence Tomography (OCT).In the recent studies made on Alzheimer's disease it is clearly investigated that are some parameter changes on the retina of the eye of the AD patients. In this research we have proposed a new scheme based on Wavelet Networks (WN) for the dimensionality reduction of OCT retinal images for the early Diagnosis of AD.
\end{abstract}

Keywords: Alzheimer's disease, OCT, early diagnosis, wavelons

Cite This Article: Sandeep C S, Sukesh Kumar A, K Mahadevan, and Manoj P, "Dimensionality Reduction of Optical Coherence Tomography Images for the Early Diagnosis of Alzheimer's Disease.” American Journal of Electrical and Electronic Engineering, vol. 5, no. 2 (2017): 58-63. doi: 10.12691/ajeee-5-2-4.

\section{Introduction}

Alzheimer's disease (AD) is the most common cause of dementia and its incidence is increasing worldwide associated with population aging [1]. AD is characterized by progressive cognitive impairment, such as memory deficit, decline in learning and executive functioning, aphasia, apraxia, agnosia and visual abnormalities [2,3]. Visual complaints are common findings in $\mathrm{AD}$ patients and these may have an important impact on autonomy and quality of life of these patients. The most common visual symptoms are impairment of spatial contrast sensitivity, motion perception, color discrimination and visual loss, which in the past, were attributed to lesions affecting the primary visual cortex and other specific areas of the brain $[4,5,6]$. Neuroimaging techniques are essential in the Diagnosis of $\mathrm{AD}$ and magnetic resonance imaging (MRI) has become the most used tool for cerebral imaging in $\mathrm{AD}$ patients, providing detailed information about brain structure. The most common findings in MRI of patients with $\mathrm{AD}$ are atrophy in the medial temporal lobe, including hippocampus, amygdala, entorhinal cortex and parahippocampal gyrus, ventricular enlargement and reduction of total brain volume [7]. Although studies have not yet completely elucidated the structural and functional changes that occur in brains of $\mathrm{AD}$ patients, some clinical and histologic studies suggest that the same neurodegenerative process that occurs in the brain, may also affect the retina, since the latter represents a peripheral part of central nervous system. Retinal pathological changes such as loss of retinal ganglion cell (RGC) and their axons were demonstrated, both in animal models and in post mortem studies of human $\mathrm{AD}$ eyes $[8,9,10,11]$. Toxic aminoacids, such as fibrillar tau and $A \beta$ aggregates were accumulated within the retina and its microvasculature, and signs of neuroinflammation were present in the retina $[12,13,14,15,16]$. Therefore, according to several clinical and histologic studies there is strong evidence of anterior visual pathway impairment in $\mathrm{AD}$ patients, with predominant involvement of RGC and their fibers $[17,18,19]$.

Optical coherence tomography (OCT) is a non-invasive technology, which acquires cross-sectional images of retinal structures allowing neural fundus integrity 
assessment. Over the last years, OCT became the most widely used technology to detect and quantify structural axonal damage in many optic nerve and neurological diseases. Axonal loss is usually quantified by measuring OCT peripapillary retinal nerve fiber layer (RNFL) that allows an indirect estimation of RGC layer impairment. Furthermore, neuronal loss can be directly accessed by estimating macular thickness measurements, since $30-35 \%$ of the retina thickness in macular area is composed by the RGCs and their fibers, as previously demonstrated in eyes with glaucoma, papilledema, compressive or demyelinating optic neuropathies $[20,21,22]$. If we take into account that the retina is considered a peripheral extension of the brain and both share similar embryological origin, it is easy to understand why OCT has become a widespread diagnostic tool in many neurological diseases. After obtaining the OCT images of AD patient, next step is to make a database of OCT AD images. For computer based assessment of OCT images we need different processes like segmentation and dimensionality reduction or feature extraction of the segmented OCT images.

\section{Related Works}

There are different ways for the segmentation and dimensionality reduction of images in the artificial intelligence field. Most prominent and popular of them are fuzzy logic and artificial neural network (ANN) approaches for segmentation of medical images [23,24]. Apart from above another promising computational intelligence method that has been widely used for various applications in different areas is wavelet network (WN).. Wavelet network takes full advantage of the characteristics of denoising, back ground reduction, recovery of the characteristic information, Neural Network capacity of universal approximation [25,26,27,28]. For this reason, it has a great ability to be used in many different applications [29,30]. For instance, in image processing wavelet networks have overcome many of the limitations in other intelligent methods such as artificial Neural Networks. The main advantage of wavelet networks over similar architectures such as multilayer perceptrons (MLP) and networks of radial basis functions (RBF) is the possibility of optimizing the wavelet network structure by means of efficient deterministic construction algorithms [31]. In this paper a specific wavelet network for segmentation of OCT images is employed. Wavelet networks are classified into two groups. They are adaptive wavelet networks (AWNs) and fixed-grid wavelet networks (FGWNs) [32]. Adaptive wavelet network is continuous wavelet transform whereas FGWN is discrete wavelet transform. Due to numerous shortcomings of AWNs like for example, complex calculations, sensitivity to initial values, the problem of measuring initial values, their application is limited [32].

In an FGWN, the outer parameters of the network like number of wavelets, scale, shift parameters value are determined. The only inner parameters of the network, weights are specified by algorithms similar to the least squares. These types of networks do not need training. In AWNs, initial values of network parameters including weights of neurons, shifts, scales of wavelets are selected randomly or using other methods. These parameters are then updated in the training stage by means of techniques such as gradient descent or back propagation (BP). Then, the optimized values of network parameters are calculated. in an FGWN, the number of wavelets, as well as the scale, shift parameters, can be determined in advance, the only unknown parameters are the weight coefficients which are calculated through methods such as least squares. So in proposed FGWN, there is no need to specify random initial values for parameters or to use gradient descent, BP, or other iterative methods. Normally, in training stage of an adaptive network, all the parameters change; on contrary, in FGWN only, the weights are specified during an on iterative process. Thus, it could be concluded that FGWNs do not need training procedure. A three-layer FGWN with one hidden layer is employed Specific wavelet network or OCT image segmentation as shown in Figure 2. The procedure for image segmentation in this paper is as follows. At first the input data are normalized. Then, after selecting a proper mother wavelet, which is usually Mexican hat due of its desirable characteristics such as convenient calculates on, adaptability to Gaussian structures, robustness against noise [33]. Next a wavelet lattice is formed. Wavelet lattice is a hyper shape of shift, scale values of wavelets. The huge dimensions of this hyper shape should be decreased, effective wavelets should be selected. All of these are accomplished through two stages of screening. This paper is unique as it employs two stages of screening. This gives ground to increase the popularity of the wavelet lattice, to estimate the function in a more accurate way which is most beneficial, significant for larger scales.

Therefore, the purpose of this article is to address the main findings on OCT in AD patients, to discuss the role of this important diagnostic tool in these patients and how OCT technology could be useful to understand morphological retinal changes in AD. The author's previous works in the area of Biomedical Engineering will definitely help to develop a new proposed tool using latest biomedical methods for the solution of the early Diagnosis of $\mathrm{AD}[1,2,3,4,5]$.

\section{Preprocessing}

Image acquisition is done by OCT camera which is shown in Figure 1; it is a kind of imaging technique used to examine the different eye related disease.

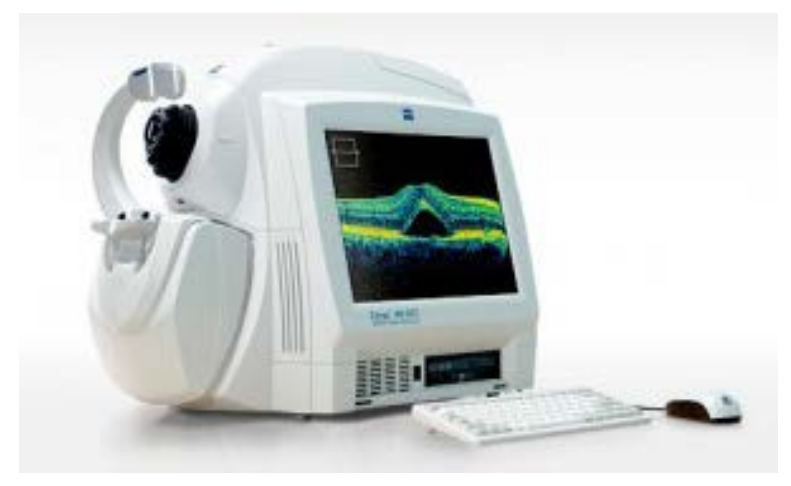

Figure 1. OCT device and OCT image on the screen 
Preprocessing is by using median filter which removes unwanted noises on the obtained OCT image of the eye. For the different steps involved in OCT image analysis of retina, segmentation is the most important stage of all. Image segmentation is defined as the process of partitioning a digital image into multiple segments. The goal of segmentation is to simplify and/or change the representation of an image into something that is more meaningful and easier to analyze. Image segmentation is mostly used to locate objects, boundaries like lines, curves etc. in images. More accurately, image segmentation is the process of assigning a label to every pixel in a digital image such that pixels with the same label share certain visual characteristics. The result of image segmentation is a set of segments that collectively cover the whole image, or a set of contours extracted from the image. Each of the pixels in a region is similar with respect to some characteristics such as such as color, intensity, or texture.

\section{Wavelet Neurons}

A wavelet can be called as a wave like oscillation with amplitude that begins at zero, increases, then decreases back to zero. A family of wavelet scan is formed from a function, called "mother wavelet," which is confined in a finite interval. "Daughter wavelets" are then formed by shifting, scaling of mother wavelet. Wavelets are mainly useful for compressing image data from a larger one. The output signal of a wavelet network with one output $f, d$ inputs and $q$ wavelons (wavelet neurons) in the hidden layer is given by

$$
\sum_{i=1}^{n} w_{i} \psi_{p i, q i}(X)=\sum_{i=1}^{n} w_{i} 2^{-p_{i} d / 2} \psi\left(2^{p i} X-q i\right)
$$

where $w_{i}, i=1,2, \ldots, n$, are weight coefficients, $\psi p i$, qi are dilated and translated versions of a mother wavelet function, $\psi$, pi, qi are scale, shift parameters, respectively [31]. A major advantage of wavelet networks over other neural architectures is the availability of efficient construction algorithms for defining the network structure [34]. In FGWN, after determining the structure, the weights $w_{i}$ in (1) can be obtained through linear estimation techniques. In this study, a constructive method is employed to build an FGWN. In many cases, input data of wavelet network vary within a wide range, this variability reduces the efficiency of wavelet network. Thus, this first stage is considered as the data pre processing stage in which the input data are normalized to a certain range in order to avoid data scattering. Here the values of R, G, B matrices of each color OCT image are mapped into $[0,1]$ range by performing normalization [35] process using:

$$
x_{n, \text { new }}^{(k)}=\frac{x_{n, o l d}^{(k)}-t_{k}}{T_{k}-t_{k}}
$$

where $x_{n \text {,new }}^{(k)}$ is the value of each colour matrix after normalization (located in $[0,1]$ range), $t_{k}$ and $T_{k}$ are minimum and maximum values of these matrices, respectively. Due to better regularities and also the ease of frame generation, multi dimensional single scaling wavelet frame is employed. Here $d$-dimensional Mexican hat radial wavelet [36] is used to implement WN. It is expressed as:

$$
\psi(x)=\eta x=\left(d-\|x\|^{\wedge} 2\right) e^{\wedge}\left(-\|x\|^{\wedge} 2 \mid 2\right) .
$$

Next step is to choose the scale and shift parameters. In this stage, minimum and maximum scale levels in the form $\left[p_{\min }, p_{\max }\right]$ and shift parameter are to be employed. A hyper shape on the wavelet parameters space that was selected in the previous stage, wavelet function is calculated for all input vectors:

$$
\psi_{p i . q j}(x)=2^{-p_{i} d / 2} \psi\left(2^{p_{i}} x-q_{j}\right) .
$$

In primary screening stage, for every scale level selected, $I_{k}$ set is formed for each input vector. In secondary screening, the shift and scale parameters of wavelets that are selected in at least two sets are determined and set $I$ is formed. Suppose that the number of selected wavelets in the last stage as L. In addition, to make the writing simpler, the couple index of $(p, q)$ is replaced with single index of $\{m=1,2,3 \ldots M\}$. In this stage, $W_{p} \times M=[\psi 1, \ldots, \psi l, \ldots, \psi M]$ matrix is calculated for all the input vectors and for all the selected shift and scale parameters that are in set $I$.

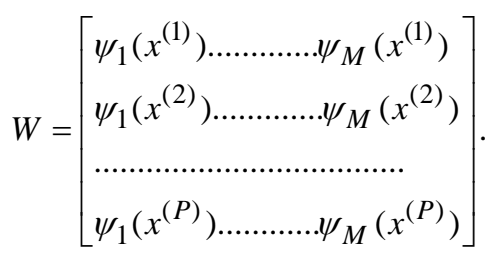

The nodes with red and blue circles are the members of sets in stage 5, respectively, and the nodes with circles in both red and blue colors are the members of set I. After two stages of screening, some of matrix members are still redundant. There as on is that only the input in formation and not the output information is taken into account for forming the wavelet matrix. A fast and efficient model structure determination approach has been implemented using the OLS algorithm. This approach has been extensively studied and widely applied in nonlinear system identification [31]. According to the OLS algorithm, to select the best subset of $W$, assuming that the size of this subset is known and denoted as $s$, the following steps should be taken. At first, the most significant wavelets in $\mathrm{W}$ is selected. Next, all other (not selected) wavelets are made orthogonal to the selected one. In the second step of the algorithm, the most significant of the remaining wavelets is again selected; then in this step, all non selected wavelets are made orthogonal with respect to the selected one, so that second selected wavelet with addition to the first one can determine the best approximation. The algorithm goes on for the rest of wavelets. Since all remaining wavelets are made orthogonal to all selected ones in each step of the algorithm, the improvement of each selectable wavelet is isolated [37]. After employing this stage, wavelet network is constructed as 


$$
f=\sum_{i=1}^{s} w_{i} \psi_{i}(x)
$$

where $s$ is the number of wavelons in the hidden layer and $w_{i}$ is the weight of wave 1 on s. After performing the OLS algorithm, $W$ is composed of ortho normal matrix $\mathrm{N}$ and upper triangular matrix $A$. So can be rewritten as:

$$
f=Q A \theta \text {. }
$$

Wavelons are the nodes creating the hidden layer of the wavelet network. By choosing the ideal number of wavelons [38], index is calculated as

$$
M S E=\frac{1}{P} \sum_{k=1}^{P}\left(\hat{f}^{(k)}-f^{(k)}\right)^{2} .
$$

Then, the number of wavelons will change until the desired error measure is achieved. After that the weight of wavelons is measured by the least-squares method.

\section{Segmentation Algorithm}

The algorithm from the previous stage is used in the present stage for segmentation of the OCT images. From images database, a number of images are randomly chosen for formation of FGWN. At first the values of R, $\mathrm{G}$, B matrices of each color OCT image are mapped into $[0,1]$ range by performing normalization process. FGWN is formed with three inputs, a hidden layer, and an output. In order to form the FGWN, the values of three colour matrices are considered as network inputs. These matrices are related to the five chosen images from the selected images for segmentation. From these images, some pixels are selected randomly. If the pixel is inside the retina layer, network output will be considered as 0 , if the pixel is outside the retina layer, the output will be considered as 1 . In this way, the FGWN is formed. After that, the value of the three matrices $\mathrm{R}, \mathrm{G}, \mathrm{B}$ for each pixel are considered as FGWN inputs, and the output of FGWN is a binary image that shows the segmented of original image in Figure 2.

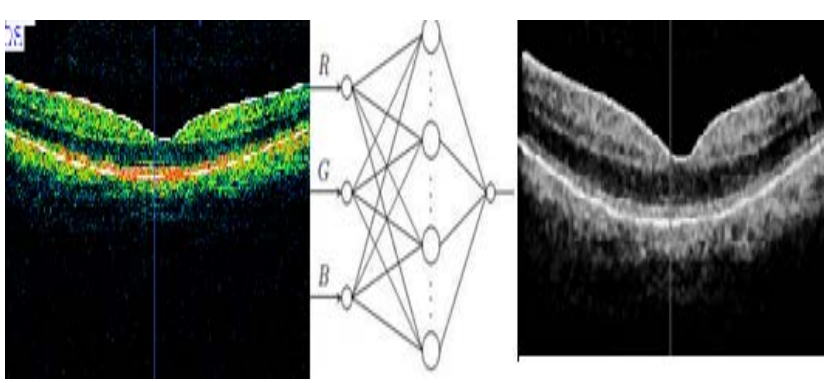

Figure 2. Presented FGWN for segmentation of OCT images

\section{Dimensionality Reduction}

After segmentation post processing is done. Since dimensionality reduction or extracting the features of retinal layers is the most essential part of diagnosing $\mathrm{AD}$, extracting the exact boundary of retinal layer is a vital task. For this, after segmentation with an FGWN the space between two shapes is filled, extra parts are eliminated, and the noise is removed. Then, the exact boundary of the layer is extracted. This is done by appropriate morphological processes, including erosion, dilation. In this paper reduction of dimensionality of OCT images are discussed below.

1) Ellipticity: a degree of similarity with the elliptical shape is obtained by dividing the area of the best fitted ellipse (largest one inside the object) by the area of the candidate object. This measure shows high values when candidate objects are normal, and low values when they are of $\mathrm{AD}$.

2) Ratio between thicknesses: considering all pairs of contour points, we divide the length of the longest path through the object by the length of the shortest path. This measure is then high for normal and low AD.

3) Curvature variance: the curvature values [39] along the object's contour present higher variation for impurities of $\mathrm{AD}$ than for control subjects. We then measure the variance of their value distribution.

4) Salience variance: the exact dilations of a contour create discrete retinal regions, wherein each contour point defines two regions composed by its closest internal and external pixels, respectively [40]. For a small dilation radius, the area of the external region is higher than the area of the internal region, when the point is convex, and the other way around is true for concave points. The salience of a point is defined as the largest area between the areas of its internal and external regions. Different from curvature, the salience is affected when opposite parts of the contour are close to each other, capturing another characteristic of the shape. The variance of its distribution produces lower values for normal, and higher values for AD.

5) Red texture: the dye solution used to enhance the layers shows them in red color. Most $\mathrm{AD}$ images are enhanced as well, but many of them are homogeneous.We capture this feature by counting the residual pixels from an area closing operation [41]. This morphological operator first fills dark regions of area less than a predefined $\lambda$ value, without affecting the object's shape, then outputs the number of pixels in the filled regions. This number is related to the degree of texture, distinguishing homogeneous (low values) from textured (higher values) objects. In our experiments, the best results were obtained with $\lambda=($ object area) $/ 4$.

6) Average number of regional minima in the gradient image inside the object: this is another way to capture texture and shapes from homogeneous objects. The regional minima are found and this measure counts the number of minima and divides it by the object's area.

7) Perimeter: this measure is used to eliminate objects with contour size out of the expected range for retinal layers.

8) Area: this measure is used to eliminate objects with area out of the expected range for retinal layers.

9) Second, third, and fourth moments: the brightness histogram of the image inside the object is quantized into 512 gray levels and we measure its variance, skewness (symmetry), and kurtosis (concentration around the mean). 


\section{Experimental Results}

The database of OCT images is taken from Sree Gokulam Medical College and Research Foundation, Trivandrum, India. For this purpose the AD patients diagnosed by the Neurologist through MRI are selected. Then those patients are forwarded to Ophthalmologist for further investigation through OCT. The dataset includes different OCT images of retina taken under the same environmental conditions. All of these images are saved as bitmap file for further processing. The size of each image was 535,974 byte; the database images employed in this paper were made free from noise or other artifacts by filtering or preprocessing stage. In case of noisy images (images which are not of desired quality or the results of segmentation are not satisfactory), or necessity of elimination of the hairs, a pre processing stage be used [36]. Among the 100 images selected for segmentation using the proposed FGWN, five images were used for building the network structure (formation of the wavelet lattice, determination of the shift scale parameters, a calculation of the network weights), and the rest were used for testing it. In our experiments, 10-12 wavelons are enough to achieve good results

Since extracting the features of retina is the most essential part of diagnosing Alzheimer's disease, extracting the retinal layer is a vital task. For this, after segmentation with an FGWN and according to the proposed algorithm, the space between two shapes is filled, extra parts are eliminated, and the noise is removed. Then, the exact boundary of retina layer is extracted. This is done by appropriate morphological processes, including erosion, dilation, closing and opening, and region filling. Size, shape, and kind of structuring elements were based on images dimensions and type of their objects that are selected tentatively and provisionally as discussed in section.

As mentioned before, segmentation is the most important and critical stage of the three stages of automatic Diagnosis of retinal layer which has a very significant role in the final outcome. Due of this reason, the performance of this state should be examined by means of appropriate criteria. Here the retinal fibre layer which is the most significant feature in detecting Alzheimer's disease is extracted by FGWN with an acceptable accuracy. Our method is quite simple and considering the satisfactory results of this study, it is very applicable for detecting $\mathrm{AD}$ by means of the computer. We have compared the segmentation process of FGWN with Neural networks (NN) and Gradient Vector Flow (GVF) as shown in Table 1. FGWN shows considerable results than NN and GVF. We have compared the different features like accuracy, precision, sensitivity and specificity. In all the circumstances FGWN is better than the other two.

Table 1. Comparison of FGWN, NN and GVF

\begin{tabular}{|c|c|c|c|c|}
\hline Method & $\begin{array}{c}\text { Accuracy } \\
\text { in \% }\end{array}$ & $\begin{array}{c}\text { Precision } \\
\text { in \% }\end{array}$ & $\begin{array}{c}\text { Sensitivity } \\
\text { in \% }\end{array}$ & $\begin{array}{c}\text { Specificity } \\
\text { in \% }\end{array}$ \\
\hline FGWN & 99.65 & 94.77 & 94.32 & 99.82 \\
\hline NN & 99.53 & 92.15 & 93.34 & 99.73 \\
\hline GVF & 98.83 & 82.28 & 83.94 & 98.83 \\
\hline
\end{tabular}

\section{Conclusion}

In this study a new approach for the segmentation as well as dimensionality reduction of OCT retina images based on fixed grid wavelet network has been employed. By comparing the retinal changes between AD and control subjects we can measure the change in retinal layer. For performing this, a wavelet lattice is formed. Parameters of wavelets are determined with two screening stages. Orthogonal least squares algorithm is used to calculate the network weights and to optimize the network structure using the developed algorithm. After that different feature of OCT images are extracted. Also the proposed method is compared with other methods, the former shows better results. Unlike NN, the results from FGWN not change in several experiments, i.e., training and testing a WN several times with the same data would lead to the same results. Therefore the proposed method provides a useful tool for the analysis of OCT retina images for predicting AD.

\section{Limitation and Future Expansion}

From the existing work we get a segmented image and features of the image are extracted. This is only the first and second screening of OCT analyses In the future works the feature selection and classification can be done. From the extracted features, best of the feature can be selected and used for classification and detection of Alzheimer's disease. In this paper we have compared FGWN with two methods In future we can compare FGWN with other techniques. For defining the network structure of a WN, an efficient constructive algorithm could be used.

\section{Acknowledgements}

The authors are thankful to Sree Gokulam Medical College and Research Foundation, Trivandrum, India for providing the necessary database of OCT images for the preparation of the paper.

\section{References}

[1] Sandeep C S, Sukesh Kumar A, A Review on the Early Diagnosis of Alzheimer's Disease (AD) through Different Tests, Techniques and Databases AMSE JOURNALS -2015-Series: Modelling C; Vol. 76; No 1 ; pp 1-22.

[2] Sandeep C S, Sukesh Kumar A, Susanth M J, "The Online Datasets Used to Classify the Different Stages for the Early Diagnosis of Alzheimer's Disease (AD)", International Journal of Engineering and Advanced Technology, Volume-6 Issue-4, April 2017: 38-45.

[3] Sandeep C S, Sukesh Kumar A, "A Psychometric Assessment Method for the Early Diagnosis of Alzheimer's disease", International Journal of Scientific \& Engineering Research IJSER, Volume 8 Issue 3-MARCH 2017.

[4] Sandeep C S, Sukesh Kumar A, Susanth M J "The Early Diagnosis of Alzheimer Disease (AD) Using CAMD, TREAD and NAAC Databases" International Journal for Science and Advance Research In Technology, IJSART - Volume 3 Issue 3 -MARCH 2017: 366-371.

[5] Sandeep C.S, Sukesh Kumar.A, "A Review Paper on the Early Diagnosis of Alzheimer's Disease(AD) through Profiling of Human Body Parameters”, Scientistlink, Coimbatore, India, 2013, 
International Journal of Computer Science and Engineering Communications (IJCSEC), Vol.1 Issue.1, pp. 21-29, December 2013.

[6] Frosch, M.P., D.C. Anthony and U.D. Girolami, 2010. The Central Nervous System. In: Robbins and Cotran Pathologic Basis of Disease, Robbins, S.L., V. Kumar, A.K. Abbas, R.S. Cotran and N. Fausto (Eds.), Elsevier srl, Philadelphia, pp: 1313-1317.

[7] Harvey, R.A., P.C. Champe, B.D. Fisher, Lippincott's Illustrated Reviews: Microbiology. 2nd Edn., Lippincott Williams and Wilkins, pp: 432, 2006.

[8] Blanks JC, Schmidt SY, Torigoe Y, Porrello KV, Hinton DR, Blanks RH. Retinal pathology in Alzheimer's disease. II. Regional neuron loss and glial changes in GCL. Neurobiol Aging. 1996; 17: 385-395.

[9] Blanks JC, Torigoe Y, Hinton DR, Blanks RH. Retinal pathology in Alzheimer's disease. I. Ganglion cell loss in foveal/parafoveal retina. Neurobiol Aging. 1996; 17: 377-384.

[10] Hinton DR, Sadun AA, Blanks JC, Miller CA. Optic-nerve degeneration in Alzheimer's disease. N Engl J Med. 1986; 315: 485-487.

[11] Sadun AA, Bassi CJ. Optic nerve damage in Alzheimer's disease. Ophthalmology. 1990; 97: 9-17.

[12] Cohen RM, Rezai-Zadeh K, Weitz TM, Rentsendorj A, Gate D, Spivak I, et al. A transgenic Alzheimer rat with plaques, tau pathology, behavioral impairment, oligomeric abeta, and frank neuronal loss. J Neurosci. 2013; 33: 6245-6256.

[13] Koronyo-Hamaoui M, Koronyo Y, Ljubimov AV, Miller CA, Ko $\mathrm{MK}$, Black KL, et al. Identification of amyloid plaques in retinas from Alzheimer's patients and noninvasive in vivo optical imaging of retinal plaques in a mouse model. Neuroimage. 2011; 54 (Suppl. 1): S204-S217.

[14] Liu B, Rasool S, Yang Z, Glabe CG, Schreiber SS, Ge J, et al. Amyloid-peptide vaccinations reduce $\beta$-amyloid plaques but exacerbate vascular deposition and inflammation in the retina of Alzheimer's transgenic mice. Am J Pathol. 2009; 175: 2099-2110.

[15] Ning A, Cui J, To E, Ashe KH, Matsubara J. Amyloid-beta deposits lead to retinal degeneration in a mouse model of Alzheimer disease. Invest Ophthalmol Vis Sci. 2008; 49: 5136-5143.

[16] Perez SE, Lumayag S, Kovacs B, Mufson EJ, Xu S. Beta-amyloid deposition and functional impairment in the retina of the APPswe/PS1DeltaE9 transgenic mouse model of Alzheimer's disease. Invest Ophthalmol Vis Sci. 2009; 50: 793-800.

[17] Curcio CA, Drucker DN. Retinal ganglion cells in Alzheimer's disease and aging. Ann Neurol. 1993; 33: 248-257.

[18] Davies DC, McCoubrie P, McDonald B, Jobst KA. Myelinated axon number in the optic nerve is unaffected by Alzheimer's disease. Br J Ophthalmol. 1995; 79: 596-600.

[19] Parisi V, Restuccia R, Fattapposta F, Mina C, Bucci MG, Pierelli F. Morphological and functional retinal impairment in Alzheimer's disease patients. Clin Neurophysiol. 2001; 112: 1860-1867.

[20] Monteiro ML, Cunha LP, Costa-Cunha LV, Maia OO, Jr, Oyamada MK. Relationship between optical coherence tomography, pattern electroretinogram and automated perimetry in eyes with temporal hemianopia from chiasmal compression. Invest Ophthalmol Vis Sci. 2009; 50: 3535-3541.

[21] Monteiro ML, Fernandes DB, Apostolos-Pereira SL, Callegaro D. Quantification of retinal neural loss in patients with neuromyelitis optica and multiple sclerosis with or without optic neuritis using Fourier-domain optical coherence tomography. Invest Ophthalmol Vis Sci. 2012; 53: 3959-3966.

[22] Monteiro ML, Afonso CL. Macular thickness measurements with frequency domain-OCT for quantification of axonal loss in chronic papilledema from pseudotumor cerebri syndrome. Eye. 2014; 28: 390-398.

[23] K.-S. Cheng, J.-S. Lin, and C.-W. Mao, "Techniques and comparative analysis of neural network systems and fuzzy systems in medical image segmentation,” Fuzzy Theor. Syst. Tech. Appl., vol. 3, pp. 973-1008, 1999.

[24] J. Jiang, P. Trundle, and J. Ren, "Medical image analysis with artificial neural networks,” Comput. Med. Imag. Graph., vol. 34, no. 8, pp. 617-631, Dec. 2010.

[25] R. M. Balabin,R. Z. Safieva, and E. I. Lomakina,“Wavelet neural network (WNN) approach for calibration model building based on gasoline near infrared (NIR) spectra," J. Chemometr. Intell. Lab. Syst., vol. 93, no. 1, pp. 58-62, Aug. 2008.

[26] Q. Zhang and A. Benveniste, "Wavelet networks," IEEE Trans. Neural Netw., vol. 3, no. 6, pp. 889-898, Nov. 1992.

[27] Y. C. Pati and P. S. Krishnaprasad, "Analysis and synthesis of feedforward neural networks using discrete affinewavelet transformations,” IEEE Trans. Neur. Netw., vol. 4, no. 1, pp. 7385, Jan. 1992.

[28] H. H. Szu, B. A. Telfer, and S. L. Kadambe, "Neural network adaptive wavelets for signal representation and classification," Opt Eng., vol. 31, no. 9, pp. 1907-1916, Sep. 1992.

[29] H. Zhang, B. Zhang, W. Huang, and Q. Tian, “Gabor wavelet associative memory for face recognition,” IEEE Trans. Neural Netw., vol. 16, no. 1, pp. 275-278, Jan. 2005.

[30] O. Jemai, M. Zaied, C. B. Amar, and M. A. Alimi, "Pyramidal hybrid approach: Wavelet network with OLS algorithm-based image classification,” Int. J. Wavel. Multir. Inf. Process., vol. 9, no. 1, pp. 111-130, Mar. 2011.

[31] R. Galvao, V. M. Becerra, and M. F. Calado, "Linear-wavelet networks,” Int. J. Appl. Math. Comput. Sci., vol. 14, no. 2, pp. 221-232, Aug. 2004.

[32] S. A. Billings and H. L. Wei, "A new class of wavelet networks for nonlinear system identification,” IEEE Trans. Neural Netw., vol. 16, no. 4, pp. 862-874, Jul. 2005.

[33] J. Gonzalez-Nuevo, F. Argueso, M. Lopez-Caniego, L. Toffolatti, J. L. Sanz, P. Vielva, and D. Herranz, "The mexican hat wavelet family.application to point source detection in CMB maps,” Mon. Not. Roy. Astron. Soc., vol. 369, pp. 1603-1610, 2006.

[34] Y. Oussar and G. Dreyfus, "Initialization by selection for wavelet network training,” Neurocomputing, vol. 34, no. 1, pp. 131-143, Sep. 2000.

[35] R. Baron and B. Girau, "Parameterized normalization: Application to wavelet networks,” in Proc. IEEE Int. Conf. Neural Netw., May 1998, vol. 2, pp. 1433-1437.

[36] Q. H. Zhang, "Using wavelet network in nonparametric estimation,” IEEE Trans. Neural Netw., vol. 8, no. 2, pp. 227-236, Mar. 1997.

[37] M. Davanipoor, M. Zekri, and F. Sheikholeslam, "Fuzzy wavelet neural network with an accelerated hybrid learning algorithm," IEEE Trans. Fuzzy Syst., vol. 20, no. 3, pp. 463-470, Jun. 2012.

[38] H. Zhou, M. Chen, L. Zou, R. Gass, L. Ferris, L. Drogowski, and J. Rehg, "Spatially constrained segmentation of dermoscopy images," in Proc. $5^{\text {th }}$ IEEE Int. Symp. Biomed. Imag.: Nano Macro, May 2008, pp. 800-803.

[39] F. Mokhtarian and S. Abbasi, "Shape similarity retrieval under affine transforms,” Pattern Recognit., vol. 35, no. 1, pp. 31-41, 2002.

[40] R. S. Torres, A. X. Falc ${ }^{a}$, and L. F. Costa, “A graph-based approach for multiscale shape analysis,” Pattern Recognit., vol. 37 no. 6, pp. 1163-1174, 2004.

[41] A. Meijster and M. H. F. Wilkinson, "A comparison of algorithms for connected set openings and closings,” IEEE Trans. Pattern Anal. Mach. Intell., vol. 24, no. 4, pp. 484-494, Apr. 2002. 